\title{
ROUGH ISOMETRIES AND DIRICHLET FINITE HARMONIC FUNCTIONS ON GRAPHS
}

\author{
PAOLO M. SOARDI
}

(Communicated by J. Marshall Ash)

\begin{abstract}
Suppose that $G_{1}$ and $G_{2}$ are roughly isometric connected graphs of bounded degree. If $G_{1}$ has no nonconstant Dirichlet finite harmonic functions, then neither has $G_{2}$.
\end{abstract}

\section{INTRODUCTION}

Suppose that $\left(X_{1}, d_{1}\right)$ and $\left(X_{2}, d_{2}\right)$ are metric spaces. A map $\phi: X_{1} \mapsto X_{2}$ is called a rough isometry (a quasi-isometry in Gromov's language [Gr]) if the two following conditions are satisfied: there are positive constants $a$ and $b$ such that, for all $x, y \in X_{1}$,

$$
a^{-1} d_{1}(x, y)-b \leq d_{2}(\phi(x), \phi(y)) \leq a d_{1}(x, y)+b
$$

there is a positive constant $c$ such that, for every $z \in X_{2}$, there exists $x \in X_{1}$ such that

$$
d_{2}(z, \phi(x)) \leq c .
$$

It is not difficult to see that rough isometry is an equivalence relation among metric spaces. Kanai (see [Ka1, Ka2]) proved that if $X_{1}$ and $X_{2}$ are roughly isometric connected Riemannian manifolds of bounded geometry, then $X_{2}$ is parabolic if $X_{1}$ is. This result was obtained by studying the relation between Dirichlet finite functions on the manifold and Dirichlet finite functions on certain graphs $(\varepsilon$-nets) on the manifold.

The discrete counterpart of Kanai's theorem was proved, among other results, in [MMT, Theorem 3.2]: if $X_{1}$ and $X_{2}$ are (the vertex sets of) two connected roughly isometric abstract graphs of bounded degree (with the usual geodesic distance on a graph), then $X_{2}$ is recurrent if $X_{1}$ is. Remember that a graph $G$ is called recurrent (transient) if the simple random walk on $G$, which assigns equal probability of passing from a vertex to any of its neighbours, is recurrent (transient); see, e.g., [G].

In this note we want to show that rough isometries between graphs preserve another important property: if $X_{1}$ and $X_{2}$ are roughly isometric connected

Received by the editors September 11,1991 and, in revised form, April 8, 1992.

1991 Mathematics Subject Classification. Primary 31C20; Secondary 94C15.

Key words and phrases. Rough isometries, infinite networks, infinite graphs, Dirichlet finite harmonic functions. 
graphs of bounded degree, and if $X_{1}$ has no nontrivial harmonic functions with finite Dirichlet sum, then neither has $X_{2}$.

This result was obtained in [MMT, Theorem 7.9] under the extra assumption that $X_{1}$ satisfies an $\varepsilon$-isoperimetric inequality $\left(0<\varepsilon \leq \frac{1}{2}\right)$. Using Yamasaki's potential theory, we can establish the theorem in full generality.

We should also mention that the nonexistence of nontrivial harmonic functions with finite Dirichlet sums is equivalent to the uniqueness of currents of finite energy in infinite resistive electrical networks; see [SW, Z]. Several criteria for existence or nonexistence of nonconstant Dirichlet finite harmonic functions are known; see [SW, T1, T2, CW].

\section{NOTATION AND PRELIMINARY RESULTS}

For every graph $G$, we will denote by $V(G)$ and $E(G)$ the vertex set and the edge set of $G$, respectively. All graphs considered in this paper will be infinite connected, without multiple edges and self-loops. Moreover, we will always assume that they are of bounded degree, i.e.,

$$
\sup _{x \in V(G)} \operatorname{deg}(x) \leq M \quad \text { for some constant } M,
$$

where $\operatorname{deg}(x)$ denotes the degree of $x \in V(G)$, i.e., its number of neighbours. The geodesic distance between two vertices $x$ and $y$ (length of a shortest path joining $x$ to $y)$ is denoted by $d_{G}(x, y)$. In particular, we will write $x \sim y$ if $x$ and $y$ are neighbours, i.e., $d_{G}(x, y)=1$.

For every graph $G$ and every positive integer $k$, the $k$-fuzz $G^{k}$ of $G$ is the graph such that $V\left(G^{k}\right)=V(G)$ and $d_{G^{k}}(x, y)=1$ if and only if $1 \leq$ $d_{G}(x, y) \leq k$. It was proved in [DS] (see also [MMT]) that $G$ is recurrent if and only if $G^{k}$ is recurrent.

Let $G_{1}$ and $G_{2}$ be graphs as above and let $\psi: V\left(G_{1}\right) \mapsto V\left(G_{2}\right)$ be an application such that $d_{G_{1}}(x, y)=1$ implies $d_{G_{2}}(\psi(x), \psi(y)) \leq 1$. Then we say that $\psi$ is a morphism from $G_{1}$ to $G_{2}$. Clearly, if $G_{1}$ is roughly isometric to $G_{2}$, i.e., if there is a rough isometry $\phi$ from $V\left(G_{1}\right)$ to $V\left(G_{2}\right)$, then $\phi$ is a morphism from $G_{1}$ to $G_{2}^{k}$, with $k \geq a+b$.

If $\psi$ is a morphism from $G_{1}$ to $G_{2}$, we define the image $G^{\prime}$ of $G_{1}$ in $G_{2}$ as follows: $V\left(G^{\prime}\right)=\psi\left(V\left(G_{1}\right)\right)$, and, for every $x, y \in V\left(G_{1}\right)$ with $x \sim y$, there is an edge $\left[x^{\prime}, y^{\prime}\right]$ with end points $x^{\prime}, y^{\prime} \in V\left(G^{\prime}\right), x^{\prime} \neq y^{\prime}$, and $\psi(x)=x^{\prime}$, $\psi(y)=y^{\prime}$. It is clear that $G^{\prime}$ is a subgraph of $G_{2}$ satisfying the assumptions made at the beginning of this section.

We will now present some results from Yamasaki's potential theory for infinite networks, which will be needed in the sequel. All the results quoted below are contained in the papers [Y1, Y2, Y3, KY].

Let $f$ be a real-valued function defined on the vertex set of a graph $G$. The Dirichlet sum of $f$ is given by

$$
D(f)=\sum_{x \sim y}|f(x)-f(y)|^{2} .
$$

If $D(f)$ is finite, we say that $f$ is Dirichlet finite. We choose a reference vertex $O$ and define a norm for Dirichlet finite functions by setting

$$
\|f\|_{\mathbf{D}(G)}^{2}=|f(o)|^{2}+D(f) .
$$


Let $\mathbf{D}(G)$ denote the space of all finite Dirichlet functions with the norm (5). It is easily seen that $\mathbf{D}(G)$ is a Hilbert space with inner product

$$
(f, g)=f(o) g(o)+\sum_{x \sim y}(f(x)-f(y))(g(x)-g(y)) .
$$

Let $\ell_{0}(G)$ denote the linear space of all real-valued finitely supported functions on $V(G)$. We denote by $\mathrm{D}_{0}(G)$ the closure of $\ell_{0}(G)$ in $\mathbf{D}(G)$.

After noticing that a graph (network) is parabolic according to Yamasaki's definition if and only if it is recurrent (see, e.g., [K, S, SY]), we have the following characterization of recurrent graphs (networks).

Proposition 1 [Y1, Theorem 3.2]. The following are equivalent:

(a) $G$ is recurrent;

(b) $1 \in \mathbf{D}_{0}(G)$;

(c) $\mathbf{D}_{0}(G)=\mathbf{D}(G)$.

Definition. A function $h$ on $V(G)$ is called harmonic if

$$
h(x)=\frac{1}{\operatorname{deg}(x)} \sum_{y \sim x} h(y) \text { for all } x \in V(G) .
$$

The subspace of all harmonic functions in $\mathbf{D}(G)$ will be denoted by $\mathbf{H D}(G)$. Yamasaki proved the discrete analogue of Royden's decomposition theorem [Y2, Lemma 1.3, Theorem 4.1].

Proposition 2. Suppose that $G$ is transient. Then, for every $f \in \mathbf{D}(G)$, there exists a unique $g \in \mathbf{D}_{0}(G)$ and a unique $h \in \mathbf{H D}(G)$ such that $f=g+h$.

We now come to the notion of extremal length of a set of paths in $G$ (see $[\mathrm{KY}, \S 2 ; \mathrm{Du}])$. Let $w$ be a nonnegative function on the edge set $E(G)$. Its energy $\mathscr{E}(w)$ is defined as

$$
\mathscr{E}(w)=\sum_{e \in E(G)} w^{2}(e)
$$

Let $\mathbf{P}$ be a set of non-self-intersecting infinite paths in $G$.

Definition. The extremal length $\lambda_{G}(\mathbf{P})$ of $\mathbf{P}$ is defined as

$$
\left(\lambda_{G}(\mathbf{P})\right)^{-1}=\inf \mathscr{E}(w),
$$

where the infimum in (7) is taken over the set of all nonnegative $w$ such that $\mathscr{E}(w)<\infty$ and such that $\sum_{e \in E(P)} w(e) \geq 1$, for every path $P \in \mathbf{P}$ (here and in the following $E(P)$ denotes the edge set of $P$ and $V(P)$ the vertex set).

If a property holds for all paths in $\mathbf{P}$, except for a subset of extremal length $\infty$, we will say that the property holds for almost all paths in $\mathbf{P}$.

Now let $\mathbf{P}_{o}$ denote the set of all one-sided non-self-intersecting infinite paths having $o$ as first vertex. Let $f \in \mathbf{D}(G)$ and $P \in \mathbf{P}_{o}$. Suppose that $\lim f(x)$ exists and is finite as $x \rightarrow \infty$ along the vertices of $P$. Then, we denote by $f(P)$ such a limit, i.e.,

$$
f(P)=\lim _{\substack{x \in V(P) \\ x \rightarrow \infty}} f(x) .
$$

We have the following results due to Kayano and Yamasaki and Yamasaki [KY, Theorem 3.1, Theorem 3.3; Y3, Theorem 3.1]. 
Proposition 3. Let $f \in \mathbf{D}(G)$. Then:

(a) for almost every $P \in \mathbf{P}_{o}, f(P)=\lim f(x)$ exists and is finite as $x \rightarrow \infty$ along the vertices of $P$;

(b) if $f \in \mathbf{D}_{0}(G)$, then $f(P)=0$ for almost every $P \in \mathbf{P}_{o}$;

(c) if $f \in \mathbf{H D}(G)$ is not a constant, then there is no constant $\gamma$ such that $f(P)=\gamma$ for almost every path $P \in \mathbf{P}_{o}$.

\section{DiRICHLET FINITE HARMONIC FUNCTIONS}

We start with a definition borrowed from Riemannian manifolds classification theory (see [Y2]).

Definition. We say that a graph $G$ belongs to the class $\mathscr{O}_{\text {HD }}$ if there is no nonconstant harmonic function on $G$ with finite Dirichlet sum.

Theorem. Suppose that $G_{1}$ and $G_{2}$ are roughly isometric infinite connected graphs of bounded degree. If $G_{1} \in \mathscr{O}_{\mathbf{H D}}$, then $G_{2} \in \mathscr{O}_{\mathbf{H D}}$.

We will split the proof in three lemmas.

Lemma 1. Suppose that $G$ is a transient connected graph satisfying (3). Then $G \in \mathscr{O}_{\mathbf{H D}}$ if and only if $G^{k} \in \mathscr{O}_{\mathbf{H D}}$.

Proof. We first prove that the norms in $\mathbf{D}(G)$ and in $\mathbf{D}\left(G^{k}\right)$ are equivalent. On the one hand, it is clear that, for every function $f$ on $V(G)=V\left(G^{k}\right)$, $\|f\|_{\mathbf{D}(G)} \leq\|f\|_{\mathbf{D}\left(G^{k}\right)}$. On the other hand, for every edge $e=\left[z_{1}, z_{2}\right] \in E\left(G^{k}\right)$, set

$$
U(e, k)=\left\{\left[x_{1}, x_{2}\right] \in E(G): d_{G}\left(x_{j}, z_{i}\right) \leq k \text { for } i, j=1,2\right\} .
$$

Clearly, every edge $[s, t] \in E(G)$ belongs to less than $M^{2(k+1)}$ sets $U(e, k)$. Now, if $d_{G^{k}}(x, y)=1$, i.e., $1 \leq d_{G}(x, y)=n \leq k$, there is a path in $G$ with vertices $x_{0}=x \sim x_{1} \sim \cdots \sim x_{n}=y$. Then

$$
\begin{aligned}
|f(x)-f(y)|^{2} & \leq k \sum_{j=1}^{n}\left|f\left(x_{j}\right)-f\left(x_{j-1}\right)\right|^{2} \\
& \leq k \sum_{[s, t] \in U([x, y], k)}|f(s)-f(t)|^{2} .
\end{aligned}
$$

It follows that

$$
\begin{aligned}
\sum_{[x, y] \in E\left(G^{k}\right)}|f(x)-f(y)|^{2} & \leq k \sum_{[x, y] \in E\left(G^{k}\right)} \sum_{[s, t] \in U([x, y], k)}|f(s)-f(t)|^{2} \\
& \leq k M^{2(k+1)} \sum_{[s, t] \in E(G)}|f(s)-f(t)|^{2}
\end{aligned}
$$

and hence,

$$
\|f\|_{\mathbf{D}\left(G^{k}\right)} \leq \sqrt{k} M^{k+1}\|f\|_{\mathbf{D}(G)} .
$$

Therefore, $\mathbf{D}(G)$ contains the same functions as $\mathbf{D}\left(G^{k}\right)$ and $\mathbf{D}_{0}(G)$ the same functions as $\mathbf{D}_{0}\left(G^{k}\right)$.

Assume that $G^{k} \in \mathscr{O}_{\mathbf{H D}}$. Let $h \in \mathbf{H D}(G)$. Since $G^{k}$ is transient together with $G$, by Proposition 2 there is a $g \in \mathrm{D}_{0}\left(G^{k}\right)$ and a constant $\kappa$ such that $h=g+\kappa$. Since $g \in \mathbf{D}_{0}(G)$ and by the uniqueness of Royden's decomposition 
in $\mathbf{D}(G), g=0$ and $h=\kappa$. Hence $G \in \mathscr{O}_{\mathbf{H D}}$. The same argument proves also the converse implication.

Lemma 2. Let $G$ and $G^{\prime}$ be transient connected graphs satisfying (3). Suppose that there is a morphism $\psi$ of $G$ onto $G^{\prime}$ in such a way that $G^{\prime}$ coincides with the image of $G$ by means of $\psi$. Moreover, suppose that there is a constant $m$ such that, for every $x, y \in V(G), \psi(x)=\psi(y)$ implies $d_{G}(x, y) \leq m$. Under these assumptions, if $G \in \mathscr{O}_{\mathbf{H D}}$, then $G^{\prime} \in \mathscr{O}_{\mathbf{H D}}$.

Proof. For every function $f^{\prime}$ on $V\left(G^{\prime}\right)$ we define a function $f$ on $V(G)$ by setting

$$
f(x)=f^{\prime}(\psi(x)) \text { for all } x \in V(G) .
$$

Let $\mathrm{C}$ denote the space of all Dirichlet finite functions on $V(G)$ of the form (8). We will show that (8) defines a Banach space isomorphism of $\mathbf{C}$ onto $\mathbf{D}\left(G^{\prime}\right)$.

We have that

$$
\begin{aligned}
\sum_{x \sim y}|f(x)-f(y)|^{2} \leq & \sum_{\substack{x^{\prime} \sim y^{\prime} \\
x^{\prime}, y^{\prime} \in V\left(G^{\prime}\right)}} \sum_{\substack{\psi(x)=x^{\prime} \\
\psi(y)=y^{\prime}}}|f(x)-f(y)|^{2} \\
& =\sum_{\substack{x^{\prime} \sim y^{\prime} \\
x^{\prime}, y^{\prime} \in V\left(G^{\prime}\right)}}\left|f^{\prime}\left(x^{\prime}\right)-f^{\prime}\left(y^{\prime}\right)\right|^{2} \sum_{\substack{x \in \psi^{-1}\left(x^{\prime}\right) \\
y \in \psi^{-1}\left(y^{\prime}\right)}} 1 .
\end{aligned}
$$

Let $s^{\prime} \in V\left(G^{\prime}\right)$ and let $s \in V(G)$ be such that $\psi(s)=s^{\prime}$. Then $\psi^{-1}\left(s^{\prime}\right) \subseteq$ $\left\{x \in V(G): d_{G}(x, s) \leq m\right\}$. Therefore, card $\psi^{-1}\left(s^{\prime}\right) \leq M^{m+1}$ and by (9) we get (choosing $o^{\prime}=\psi(o)$ )

$$
D(f) \leq M^{2(m+1)} D\left(f^{\prime}\right), \quad \text { whence }\|f\|_{\mathbf{D}(G)} \leq M^{m+1}\left\|f^{\prime}\right\|_{\mathbf{D}\left(G^{\prime}\right)} .
$$

On the other hand, since $G^{\prime}$ coincides with the image of $G$, for every $x^{\prime}, y^{\prime} \in$ $V\left(G^{\prime}\right), x^{\prime} \sim y^{\prime}$, there exist $x, y \in V(G)$ such that $x \sim y$ and $\psi(x)=x^{\prime}$, $\psi(y)=y^{\prime}$. It follows that

$$
D\left(f^{\prime}\right) \leq D(f), \quad \text { whence }\left\|f^{\prime}\right\|_{\mathbf{D}\left(G^{\prime}\right)} \leq\|f\|_{\mathbf{D}(G)} .
$$

Now we claim that, if $f$ and $f^{\prime}$ are as in (8), $f \in \mathbf{C} \cap \mathbf{D}_{0}(G)$ if and only if $f^{\prime} \in \mathbf{D}_{0}\left(G^{\prime}\right)$.

One implication is obvious by (10). To prove the converse, write $V(G)$ as the disjoint union of the classes $\psi^{-1}\left(x^{\prime}\right)$ with $x^{\prime} \in V\left(G^{\prime}\right)$. For every $x \in V(G)$ let $\{x\}$ be the class of $x$ and let $\omega(x)=\operatorname{card}\{x\}$. For every $g \in \mathbf{D}(G)$ we define the mean $\mathscr{M} g$ of $g$ by

$$
\mathscr{M} g(x)=\frac{1}{\omega(x)} \sum_{s \in\{x\}} g(s) \text { for all } x \in V(G) .
$$

Suppose that $x \sim y$. Then

$$
\begin{aligned}
|\mathscr{M} g(x)-\mathscr{M} g(y)|^{2} & =(\omega(x) \omega(y))^{-2}\left(\sum_{\substack{s \in\{x\} \\
t \in\{y\}}} g(s)-g(t)\right)^{2} \\
& \leq(\omega(x) \omega(y))^{-1} \sum_{\substack{s \in\{x\} \\
t \in\{y\}}}|g(s)-g(t)|^{2} .
\end{aligned}
$$


Clearly, $d_{G}(s, t) \leq 2 m+1$ for every $s \in\{x\}$ and $t \in\{y\}$. Hence, there exists a path with vertices $s=x_{0} \sim x_{1} \sim \cdots \sim x_{n}=t$ such that $n \leq 2 m+1$. It follows that

$$
|g(s)-g(t)|^{2} \leq(2 m+1) \sum_{j=1}^{n}\left|g\left(x_{j}\right)-g\left(x_{j-1}\right)\right|^{2} .
$$

We set, for every couple of vertices $z_{1}, z_{2}$ in $V(G)$ such that $d_{G}\left(z_{1}, z_{2}\right) \leq$ $2 m+1$,

$$
N\left(z_{1}, z_{2}\right)=\left\{\left[x_{1}, x_{2}\right] \in E(G): d_{G}\left(x_{j}, z_{i}\right) \leq 2 m+1 \text { for } i, j=1,2\right\} .
$$

Every edge $\left[x_{1}, x_{2}\right] \in E(G)$ belongs to less than $M^{4(m+1)}$ sets $N\left(z_{1}, z_{2}\right)$ so that by (13) above,

$$
|g(s)-g(t)|^{2} \leq(2 m+1) \sum_{\left[x_{1}, x_{2}\right] \in N(s, t)}\left|g\left(x_{1}\right)-g\left(x_{2}\right)\right|^{2} .
$$

Taking (12) into account,

$$
\begin{aligned}
& \sum_{x \sim y}|\mathscr{M} g(x)-\mathscr{M} g(y)|^{2} \leq(2 m+1) \sum_{\substack{x \sim y \\
\sum_{s} \in\{x\} \\
t \in\{y\}}} \sum_{\left[x_{1}, x_{2}\right] \in N(s, t)}\left|g\left(x_{1}\right)-g\left(x_{2}\right)\right|^{2} \\
& \leq(2 m+1) M^{4(m+1)} \sum_{\left[x_{1}, x_{2}\right] \in E(G)}\left|g\left(x_{1}\right)-g\left(x_{2}\right)\right|^{2} .
\end{aligned}
$$

Hence,

$$
D(\mathscr{M} g) \leq(2 m+1) M^{4 m+4} D(g) .
$$

Moreover, it is easy to see that

$$
|g(s)|^{2} \leq(m+1)\left(D(g)+|g(o)|^{2}\right) \text { for every } s \in\{o\} .
$$

Hence,

$$
|\mathscr{M} g(o)|^{2} \leq \frac{1}{\omega(o)} \sum_{s \in\{o\}}|g(s)|^{2} \leq(m+1)\|g\|_{\mathbf{D}(G)}^{2} .
$$

Finally, from (14) and (16) we get

$$
\|\mathscr{M} g\|_{\mathbf{D}(G)} \leq \sqrt{3 m+2} M^{2 m+2}\|g\|_{\mathbf{D}(G)} .
$$

Assume that $f$ is a function in $\mathbf{C} \cap \mathbf{D}_{0}(G)$. Let $f_{n} \in \ell_{0}(G)$ be a sequence such that $\left\|f-f_{n}\right\|_{\mathbf{D}(G)} \rightarrow 0$. Since $\mathscr{M} f=f,(17)$ implies $\left\|f-\mathscr{M} f_{n}\right\|_{\mathbf{D}(G)} \rightarrow 0$. Therefore, our claim follows from (11).

Suppose now that $f^{\prime} \in \mathbf{H D}\left(G^{\prime}\right)$. Since $G$ is transient, by Proposition 2 there is a constant $\kappa$ and a function $g \in \mathbf{D}_{0}(G)$ such that $f=g+\kappa$. If $g$ and $g^{\prime}$ are related as $f$ and $f^{\prime}$ in (8), we have that $g^{\prime} \in \mathbf{D}_{0}\left(G^{\prime}\right)$ and $f^{\prime}=g^{\prime}+\kappa$. By the uniqueness of Royden's decomposition in $\mathbf{D}\left(G^{\prime}\right), g^{\prime}=0$ and $f^{\prime}=\kappa$. Hence, $G^{\prime} \in \mathscr{O}_{\text {HD }}$.

Lemma 3. Let $G$ be a transient connected subgraph of a connected graph $H$ satisfying (3). Assume that the inclusion map of $G$ into $H$ is a rough isometry. If $G \in \mathscr{O}_{\mathbf{H D}}$, then $H \in \mathscr{O}_{\mathbf{H D}}$.

Proof. Let $f \in \mathbf{H D}(H)$. We will show that there is a constant $\gamma$ such that, for almost all paths $P \in \mathbf{P}_{o}, f(x)$ tends to $\gamma$ as $x \rightarrow \infty$ along the vertices of $P$. 
By Proposition 3(c) this will imply that $f$ is a constant, thus proving the thesis of the lemma.

Let $g$ denote the restriction of $f$ to $V(G)$. Clearly, $g \in \mathbf{D}(G)$. Let $\mathbf{P}_{o}^{G}$ denote the set of all infinite paths in $\mathbf{P}_{o}$, all of whose edges are in $E(G)$ (we may assume $o \in V(G))$. Since $G \in \mathscr{O}_{\mathbf{H D}}$, by Proposition 2 and Proposition 3(b), there exists a constant $\gamma$ such that $g(P)=\gamma$, for almost every path $P$ in $\mathbf{P}_{o}^{G}$. We set

$$
\begin{aligned}
& \mathbf{P}^{\mathrm{I}}=\left\{P \in \mathbf{P}_{o}: f(P)=\gamma\right\}, \\
& \mathbf{P}^{\mathrm{II}}=\left\{P \in \mathbf{P}_{o}: f(P) \neq \gamma\right\}, \\
& \mathbf{P}^{\mathrm{III}}=\left\{P \in \mathbf{P}_{o}: f(P) \text { does not exist }\right\}, \\
& \mathbf{Q}=\mathbf{P}^{\mathrm{II}} \cap \mathbf{P}_{o}^{G} .
\end{aligned}
$$

Therefore almost every path $P \in \mathbf{P}_{o}^{G}$ belongs to $\mathbf{P}^{\mathrm{I}}$. Moreover, $\lambda_{H}\left(\mathbf{P}^{\mathrm{III}}\right)=\infty$ by Proposition 3(a). To prove the lemma we have only to show that $\lambda_{H}\left(\mathbf{P}^{\mathrm{II}}\right)=$ $\infty$ (note that $H$ is transient so that, by [Y1, Theorem 4.1], $\lambda_{H}\left(\mathbf{P}_{o}\right)<\infty$ ).

In what follows, we will write $x \sim y$ if $d_{H}(x, y)=1$ and $x \approx y$ if $d_{G}(x, y)=1$. Now, we will associate to every $P \in \mathbf{P}^{\mathrm{II}}$ a path $\widetilde{P}$ in $\mathbf{Q}$. Choose any path $P \in \mathbf{P}^{\mathrm{II}}$ with distinct vertices $o=x_{0} \sim x_{1} \sim \cdots \sim x_{n} \sim \cdots$. By (2), for every $n$ there exist vertices $y_{n} \in V(G)$ (not necessarily distinct) such that $d_{H}\left(x_{n}, y_{n}\right) \leq c$. Let $\rho=a(2 c+1+b)$, where $a, b, c$ are as in (1), (2). We have by (1)

$$
\begin{aligned}
d_{G}\left(y_{n}, y_{n+1}\right) & \leq a d_{H}\left(y_{n}, y_{n+1}\right)+a b \\
& \leq a\left(d_{H}\left(y_{n}, x_{n}\right)+d_{H}\left(x_{n}, x_{n+1}\right)+d_{H}\left(x_{n+1}, y_{n+1}\right)\right)+a b \\
& \leq a(2 c+1+b)=\rho .
\end{aligned}
$$

Note also that $d_{H}\left(y_{n}, y_{n+1}\right) \leq d_{G}\left(y_{n}, y_{n+1}\right)$.

Suppose $y_{n} \neq y_{n+1}$. By (18), there are distinct vertices $z_{r} \in V(G), r=$ $0, \ldots, k$, with $k \leq \rho$, such that

$$
y_{n}=z_{0} \approx z_{1} \approx \cdots \approx z_{k}=y_{n+1} .
$$

It follows that there exists a path $\widetilde{P} \in \mathbf{P}_{o}^{G}$ with distinct vertices $t_{j} \in V(G)$,

$$
o=t_{0} \approx t_{1} \approx \cdots \approx t_{j} \approx \cdots
$$

and a nondecreasing sequence of subscripts $j(n) \rightarrow \infty$ such that

$$
t_{j(n)}=y_{n}, \quad j(n+1)-j(n) \leq \rho, \quad d_{H}\left(x_{n}, t_{j(n)}\right) \leq c .
$$

Note that, for every integer $r$, the cardinality of the set $\{n: j(n)=r\}$ is smaller than $M^{c+1}$.

For every $n$ there are vertices $v_{r} \in V(H), r=0, \ldots, m$, with $m \leq c$, such that

$$
v_{0}=x_{n} \sim v_{1} \sim \cdots \sim v_{m}=t_{j(n)} .
$$

Then, as $n \rightarrow \infty$,

$$
\left|f\left(x_{n}\right)-f\left(t_{j(n)}\right)\right|^{2} \leq c \sum_{r=1}^{m}\left|f\left(v_{r}\right)-f\left(v_{r-1}\right)\right|^{2} \rightarrow 0
$$


so that $g\left(t_{j(n)}\right)=f\left(t_{j(n)}\right) \rightarrow f(P) \neq \gamma$. In the same way, we see that the whole sequence $g\left(t_{j}\right)$ tends to $f(P)$ since, for $j(n)<j<j(n+1)$, we have

$$
\left|f\left(t_{j}\right)-f\left(t_{j(n)}\right)\right|^{2} \leq \rho \sum_{r=1}^{k}\left|f\left(z_{r}\right)-f\left(z_{r-1}\right)\right|^{2} \rightarrow 0 .
$$

It follows that the path $\widetilde{P}$ with vertices $t_{j}$ belongs to $\mathbf{Q}$.

Since $\lambda_{G}(\mathbf{Q})=\infty$, by the necessary and sufficient condition for a set of paths to have infinite extremal length [KY, Lemma 2.3], there exists a positive function $w$ on $E(G)$ such that

$$
\begin{gathered}
\sum_{\tilde{e} \in E(G)} w^{2}(\tilde{e})=\mathscr{E}(w)<\infty \\
\sum_{\tilde{e} \in E(P)} w(\tilde{\boldsymbol{e}})=\infty \quad \text { for all } P \in \mathbf{Q} .
\end{gathered}
$$

Let $\rho$ be as above and set $\delta=\rho+c$. For every edge $e=\left[z_{1}, z_{2}\right] \in E(H)$, set

$$
U(e, \delta)=\left\{\tilde{e}=\left[x_{1}, x_{2}\right] \in E(G): d_{H}\left(x_{j}, z_{i}\right) \leq \delta \text { for } i, j=1,2\right\} .
$$

We define $w^{*}$ on $E(H)$ in the following way:

$$
w^{*}(e)=\sup _{\tilde{e} \in U(e, \delta)} w(\tilde{e}) \quad \text { for all } e \in E(H) .
$$

Clearly, $w^{* 2}(e) \leq \sum_{\tilde{e} \in U(e, \delta)} w^{2}(\tilde{e})$ for all $e \in E(H)$. Moreover, every edge $\tilde{e} \in E(G)$ belongs to less than $M^{2(\delta+1)}$ sets $U(e, \delta), e \in E(H)$. Therefore, (20) gives

$$
\begin{aligned}
\mathscr{E}\left(w^{*}\right) & =\sum_{e \in E(H)} w^{* 2}(e) \leq \sum_{e \in E(H)} \sum_{\tilde{e} \in U(e, \delta)} w^{2}(\tilde{e}) \\
& \leq M^{2(\delta+1)} \sum_{\tilde{e} \in E(G)} w^{2}(\tilde{e})<\infty
\end{aligned}
$$

We now claim that

$$
\sum_{e \in E(P)} w^{*}(e)=\infty \text { for all } P \in \mathbf{P}^{\mathrm{II}}
$$

Let, for every $P \in \mathbf{P}^{I I}$ with vertices $x_{n}, \widetilde{P}$ be the path in $\mathbf{Q}$ with vertices $t_{j}$ constructed above. For such a $\widetilde{P}, \sum_{\tilde{e} \in E(\widetilde{P})} w(\tilde{\boldsymbol{e}})=\infty$, by (21). For every edge $\left[x_{n}, x_{n+1}\right] \in E(P)$, such that $j(n) \neq j(n+1)$,

$$
\rho w^{*}\left(\left[x_{n}, x_{n+1}\right]\right) \geq \sum_{j=j(n)+1}^{j(n+1)} w\left(\left[t_{j-1}, t_{j}\right]\right)
$$

since, by (18) and (19), $\left[t_{j-1}, t_{j}\right] \in U\left(\left[x_{n}, x_{n+1}\right], \delta\right)$ for $j=j(n)+1, \ldots$, $j(n+1)$.

It follows, from (21) and (24), that

$$
\sum_{e \in E(P)} w^{*}(e) \geq \rho^{-1} \sum_{\tilde{e} \in E(\widetilde{P})} w(\tilde{e})=\infty
$$


Therefore, by (22), (23), and [KY, Lemma 2.3] again, $\lambda_{H}\left(P^{\mathrm{II}}\right)=\infty$. Hence, $f(P)=\gamma$ for almost every path in $\mathbf{P}_{o}$.

Proof of the theorem. If $G_{1}$ and $G_{2}$ are recurrent, then they belong to $\mathscr{O}_{\text {HD }}$ by [Y2, Theorem 6.1]. So let us assume that they are transient. Let $\phi: V\left(G_{1}\right) \mapsto$ $V\left(G_{2}\right)$ be the rough isometry. Let $k>a+b$. Then $\phi$ is a morphism from $G_{1}$ to $G_{2}^{k}$. Let $G_{1}^{\prime}$ be the image of $G_{1}$ in $G_{2}^{k}$ by means of $\phi$. By Lemma 2 (with $m=a b$, by (1)), $G_{1}^{\prime}$ belongs to $\mathscr{O}_{\text {HD }}$. By Lemma $3, G_{2}^{k}$ belongs to $\mathscr{O}_{\text {HD }}$. Finally, by Lemma $1, G_{2}$ belongs to $\mathscr{O}_{\text {HD }}$.

Remark. Let $1<p<\infty$. According to Yamasaki [Y1], the Dirichlet sum of order $p$ of a real-valued function $f$ on the vertex set of a graph $G$ is defined as

$$
D^{(p)}(f)=\sum_{x \sim y}|f(x)-f(y)|^{p}
$$

Moreover, $f$ is called $p$-harmonic if it satisfies the following equation for every $x \in V(G)$ :

$$
\sum_{x \sim y} \operatorname{sign}(f(x)-f(y))|f(x)-f(y)|^{p-1}=0 .
$$

Using Yamasaki's potential theory and the same arguments as in this paper, it is not difficult to see that our main result holds true also in the case of $p$-harmonic functions. Namely, if $G_{1}$ and $G_{2}$ are roughly isometric connected graphs of bounded degree, and if $G_{1}$ has no nonconstant $p$-harmonic functions with finite Dirichlet sum of order $p$ (see, e.g., [SY]), then $G_{2}$ has the same property.

\section{REFERENCES}

[CW] D. I. Cartwright and W. Woess, Infinite graphs with nonconstant Dirichlet finite harmonic functions, SIAM J. Discrete Math. 3 (1992), 380-385.

[DS] P. G. Doyle and J. L. Snell, Random walks and electrical networks, Math. Assoc. Amer., Washington, D.C., 1984.

[Du] R. J. Duffin, The extremal length of a network, J. Math. Anal. Appl. 5 (1962), 200-215.

[G] P. Gerl, Random walks on graphs, Probability Measures on Groups VII (H. Heyer, ed.), Lecture Notes in Math., vol. 1210, Springer, New York, 1985, pp. 285-303.

[Gr] M. Gromov, Hyperbolic groups, Essays in Group Theory (S. M. Gersten, ed.), Springer, New York, 1987, pp. 75-263.

[K] V. A. Kaimanovich, Dirichlet norms, capacities and generalized isoperimetric inequalities for Markov operators, Potential Anal. 1 (1992), 61-82.

[Ka1] M. Kanai, Rough isometries and combinatorial approximation of geometries of non-compact Riemannian manifolds, J. Math. Soc. Japan 37 (1985), 391-413.

[Ka2] _ Rough isometries and the parabolicity of Riemannian manifolds, J. Math. Soc. Japan 38 (1986), 227-238.

[KY] T. Kayano and M. Yamasaki, Boundary limits of discrete Dirichlet potentials, Hiroshima J. Math. 14 (1984), 401-406.

[MMT] S. Markvorsen, S. Mc Guinness, and C. Thomassen, Transient random walks on graphs and metric spaces with applications to hyperbolic surfaces, Proc. London Math. Soc. 64 (1992), $1-20$.

[S] P. M. Soardi, Recurrence and transience of the edge graph of a tiling of the euclidean plane, Math. Ann. 287 (1990), 613-626. 
[SW] P. M. Soardi and W. Woess, Uniqueness of currents in infinite resistive networks, Discrete Appl. Math. 31 (1991), 37-49.

[SY] P. M. Soardi and M. Yamasaki, Classification of infinite networks and its applications, Circuits Systems Signal Process. 12 (1993), 133-149.

[T1] C. Thomassen, Resistances and currents in infinite electrical networks, J. Combin. Theory Ser. B 49 (1990), 87-102.

[T2] Isoperimetric inequalities and transient random walks on graphs (to appear).

[Y1] M. Yamasaki, Parabolic and hyperbolic infinite networks, Hiroshima Math. J. 7 (1977), 135-146.

[Y2] - , Discrete potentials on an infinite network, Mem. Fac. Sci. Shimane Univ. 13 (1979), 31-44.

[Y3] I I _ Ieal boundary limit of discrete Dirichlet functions, Hiroshima Math. J. 16 (1986), 353-360.

[Z] A. H. Zemanian, Infinite electrical networks, Proc. IEEE 64 (1976), 6-17.

Dipartimento di Matematica dell'Università, via Saldini 50, 20133 Milano, Italy

E-mail address: soardievmimat .mat .unimi.it 